\title{
Cell-Centered Multigrid for Interface Problems
}

\author{
P. WESSELING
}

\author{
Department of Mathematics and Informatics, Delft University of Technology, \\ P.O. Box 356, 2600 AJ Delfi, The Netherlands
}

Received June 12, 1987; revised November 18, 1987

\begin{abstract}
A multigrid method is presented for cell-centered discretizations of elliptic partial differential equations. The method works both for smooth and strongly discontinuous coefficients, even though, in contrast with earlier works, the prolongation and restriction operators do not depend on the equation. 1988 Academic Press, Inc.
\end{abstract}

\section{INTRODUCTION}

The multigrid method to be presented will be developed for the equation

$$
\begin{aligned}
-\frac{\partial}{\partial x}\left(a \frac{\partial \phi}{\partial x}\right)-\frac{\partial}{\partial y}\left(a \frac{\partial \phi}{\partial y}\right) & =f, \\
(x, y) \in \Omega & =(0,1) \times(0,1),\left.\quad \phi\right|_{a \Omega}=g, \quad a>0 .
\end{aligned}
$$

The coefficient $a(x, y)$ is not continuous everywhere. This precludes application of standard multigrid methods. Alcouffe et al. [1], Dendy [3], Kettler and Meijerink [6] (see also Kettler [7]) have developed special multigrid methods that work well for the problem considered here. In these methods the prolongation and restricton operators depend on the discrete approximation to (1.1). Until now, theoretical justification is lacking and seems hard to come by. In the following, a multigrid method is proposed for (1.1) that also works in practice, is simpler, and can be justified theoretically. The difference with the methods just mentioned is that prolongation and restriction are not problem-dependent and that grid coarsening is done cell-wise rather than point-wise. What this means will be made clear in the sequel.

\section{Finite Volume Discretization}

For convenience, the mesh size will be $h$ in both directions. The domain $\Omega$ is subdivided in finite volumes or cells, which are squares of size $h$, with centers at the points

$$
\Omega_{h}=\left\{(x, y): x=x_{i}=\left(i-\frac{1}{2}\right) h, y=y_{j}=\left(j-\frac{1}{2}\right) h ; i, j=1,2, \ldots, n ; h=1 / n\right\} .
$$


The cell with center at $\left(x_{i}, y_{j}\right)$ is denoted by $\Omega_{i j}$, and $\phi_{i j}$ is the value of $\phi$ at the center. Often, this is called a block-centered or cell-centered grid. Forward and backward divided differences in $x$ - and $y$-direction are defined by

$$
d_{x} \phi_{i j}=\left(\phi_{i+1, j}-\phi_{i j}\right) / h, \quad \nabla_{x} \phi_{i j}=\left(\phi_{i j}-\phi_{i-1, j}\right) / h,
$$

and similarly for $\Delta_{y}$ and $\nabla_{y}$ :

For completeness we briefly review the elementary aspects of finite volume discretization of (1.1) with discontinuous coefficient $a$. Equation (1.1) is integrated over the finite volume $\Omega_{i j}$. With the Gauss divergence theorem this results in

$$
-\int_{\partial \Omega_{i j}} a \phi_{, \alpha} n_{\alpha} d \Gamma=\int_{\Omega_{i j}} f d \Omega=h^{2} f_{i j}
$$

with the summation convention for the index $\alpha$.

Let $S_{i j}^{z}$ be the side of $\Omega_{i j}$ with outward normal in the $x_{x}$-direction $\left(x_{1}=x, x_{2}=y^{\prime}\right)$. Equation (2.3) can be rewritten as

$$
-\nabla_{\alpha} F_{\alpha}^{i j}=h f_{i j}
$$

with the flux $F_{\alpha}^{i j}$ defined as

$$
F_{\alpha}^{i j}=\int_{S_{i j}^{z}} a \phi,_{,} d \Gamma
$$

We discuss the approximation of $F_{1}^{i j} ; F_{2}^{i j}$ is treated similarly. $F_{1}^{i}$ is approximated as

$$
F_{1}^{i j} \simeq h a_{i j} \frac{\partial \phi}{\partial x}\left(x_{i}+h / 2, y_{i}\right)
$$

where $a_{i j}$ is the average of $a$ over $\Omega_{i j}$. The approximation

$$
\frac{\partial \phi}{\partial x}\left(x_{i j}+h / 2, y_{i j}\right) \simeq \Delta_{\mathrm{r}} \phi_{i j}
$$

is out of the question, since $a_{i j}$ may differ strongly between adjacent cells, so that $\partial \phi / \partial x$ may have large jumps at cell boundaries. A correct itpproximation is obtained as follows. Point of departure is that $\phi$ and a $i \phi i x$ are continuous. Denote for brevity $\phi\left(x_{i j}+h / 2, y_{i j}\right)$ by $\phi^{*}$. Then we approximate $F_{1}^{\prime \prime}$ by

$$
F_{\mathrm{I}}^{i j} \simeq 2 a_{i j}\left(\phi^{*}-\phi_{i j}\right)=2 a_{i+1 . j}\left(\phi_{i+1.1}-\phi^{*}\right) .
$$

Elimination of $\phi^{*}$ from (2.8) results in

$$
F_{1}^{i j} \simeq h w_{i j}^{*} \Delta_{1} \phi_{11}
$$


with

$$
w_{i j}^{x}=2 a_{i j} a_{i+1, j} /\left(a_{i j}+a_{i+1, j}\right) .
$$

Similarly, we obtain

$$
F_{2}^{i j} \simeq h w_{i j}^{v} \Delta_{y} \phi_{i j}
$$

with

$$
w_{i j}^{v}=2 a_{i j} a_{i, j+1} /\left(a_{i j}+a_{i, j+1}\right) .
$$

Substitution of (2.9) and (2.11) in (2.4) results in

$$
-\left(\nabla_{x} w^{x} \Delta_{x}+\nabla_{y} w^{y} \Delta_{y}\right) \phi=f .
$$

It is easy to see that $w^{x}$ and $w^{y}$ satisfy

$$
\inf (a) \leqslant w^{x}, w^{y} \leqslant \sup (a) .
$$

The Dirichlet boundary condition is implemented as follows. Consider the side $x=0$. There $F_{1}^{(i)}$ is approximated by (cf. (2.8)):

$$
F_{1}^{0 j} \simeq 2 a_{1 j}\left(\phi_{1 j}-g_{j}\right) .
$$

A Neumann boundary condition gives $F_{1}^{0 j}$ directly.

\section{Prolongation and Restriction}

The reader is assumed to be familiar with multigrid methods. For an introduction, see, for example, Hackbusch and Trottenberg [4], Hackbusch [5], or McCormick [8].

Coarse grids are constructed cell-wise. That is, coarser grids $\Omega_{2 h}, \Omega_{4 h}, \ldots$ are obtained by successively doubling $h$ in (2.1). Hence, each coarse cell is the union of four finer cells. The cell centers of a coarse grid do not belong to the next finer grid. This is different from point-wise coarsening, where coarse grids are constructed by deleting grid points, so that coarse grid points always belong to a finer grid.

The grid with mesh size $h$ is denoted by $\Omega_{h}$, and $\Phi_{h}: \Omega_{h} \rightarrow \mathbb{R}$ is the corresponding set of grid functions. Elements of $\Phi_{h}$ are denoted by $\phi^{h}, \psi^{h}$.

In this section the choice of prolongation and restriction operators

$$
P_{h}: \Phi_{2 h} \rightarrow \Phi_{h}, \quad R_{2 h}: \Phi_{h} \rightarrow \Phi_{2 h}
$$

is discussed. One possibility is

$$
\begin{aligned}
\left(P_{h} \phi^{2 h}\right)_{2 i, 2 j} & =\left(P_{h} \phi^{2 h}\right)_{2 i-1,2 j}=\left(P_{h} \phi^{2 h}\right)_{2 i, 2 j-1} \\
& =\left(P_{h} \phi^{2 h}\right)_{2 i-1,2 j-1}=\phi_{i j}^{2 h} .
\end{aligned}
$$


A possibility for $R_{2 h}$ is

$$
R_{2 h}=P_{h}^{*},
$$

with superscript $*$ denoting the adjoint. With the inner product

$$
\left(\phi^{h}, \psi^{h}\right)=h^{2} \sum_{\Omega_{h}} \phi_{i j}^{h} \psi_{i j}^{h}
$$

we find that the stencil of $R_{2 n}$ defined by (3.1), (3.2) is

$$
\left[R_{2 h}\right]=\frac{1}{4}\left[\begin{array}{ll}
1 & 1 \\
1 & 1
\end{array}\right]
$$

where $[\cdot]$ denotes the stencil of the corresponding operator.

$P_{h}$ and $R_{2 h}^{*}$ interpolate polynomials exactly of degree at most 0 . Their order $m_{p}$, $m_{R}$ is defined to be the maximum degree of exactly interpolated polynomials plus 1 : hence for (3.1), (3.2) we have

$$
m_{P}=m_{R}=1 .
$$

We must have

$$
m_{P}+m_{R}>2 m
$$

(Brandt [2], Hackbusch [5]), with $2 m$ the order of the differential equation to be solved. Hence, (3.1), (3.2) are not right for (1.1). See Wesseling [10] for what happens when one does use (3.1), (3.2) for (1.1).

A restriction with $m_{R}=2$ is given by

$$
\left[R_{2 h}\right]=\frac{1}{16}\left[\begin{array}{llll}
1 & 1 & 0 & 0 \\
1 & 3 & 2 & 0 \\
0 & 2 & 3 & 1 \\
0 & 0 & 1 & 1
\end{array}\right]
$$

At the boundaries, (3.7) has to be modified. For a Dirichlet boundary condition we obtain at the boundary $y=1$ or at the boundary $x=0$,

$$
\left[R_{2 h}\right]=\frac{1}{16}\left[\begin{array}{cccc}
0 & 0 & 0 & 0 \\
0 & 2 & 2 & 0 \\
0 & 2 & 3 & 1 \\
0 & 0 & 1 & 1
\end{array}\right]
$$

and similarly at other parts of the boundary. This restriction is obtained as adjoint of linear interpolation. For simplicity, (3.8) is also used in the case of Neumann boundary conditions. 
Let the system of equations to be solved on $\Omega_{h}$ be denoted as

$$
A_{h} \phi^{h}=f^{h} \text {. }
$$

On $\Omega_{2 h}, A_{h}$ is approximated by

$$
A_{2 h}=R_{2 h} A_{h} P_{h}
$$

Let $A_{h}$ have a 7-point stencil:

$$
\left[A_{h}\right]=\left[\begin{array}{lll}
* & * & 0 \\
* & * & * \\
0 & * & *
\end{array}\right] .
$$

Then it is found that with $P_{h}, R_{2 h}$ given by (3.1) and (3.7)-(3.8), $A_{2 h}$ as given by (3.10) also has a 7-point stencil. This is also true if $P_{h}^{*}$ is given by (3.7)-(3.8) and $R_{2 h}$ by (3.4). However, if both $P_{h}^{*}$ and $R_{2 h}$ are given by (3.7)-(3.8) then the stencil of $A_{2 h}$ is larger than that of $A_{h}$. Therefore it was decided to choose $R_{2 h}$ according to (3.7)-(3.8) and $P_{h}$ according to (3.1). Note that we have $m_{P}+m_{R}=3$, which suffices.

It is easy to obtain $A_{2 h}$ explicitly from (3.10), with the choice just made for $P_{h}$ and $R_{2 h}$. It is found that $A_{2 / t}$ corresponds to the following discrete equation on the coarse grid (cf. Eq. (2.13)),

$$
-\left(\bar{\nabla}_{x} \bar{w}^{x} \bar{\Delta}_{x}+\bar{\nabla}_{y} \bar{w}^{y} \bar{J}_{y}\right) \bar{\phi}=\vec{f},
$$

where quantities belonging to the coarse grid are denoted by an overbar. We find the simple relation for $\bar{w}^{x}, \bar{w}^{y}$,

$$
\bar{w}_{i j}^{x}=\frac{1}{2}\left(w_{2 i, 2 j}^{x}+w_{2 i, 2 j-1}^{x}\right), \quad \bar{w}_{i j}^{j}=\frac{1}{2}\left(w_{2 i, 2 j}^{k}+w_{2 i-1,2 j}^{v}\right) .
$$

Hence, in this case construction of coarse grid matrices by (3.10) (Galerkin approximation) is extremely cheap. Note that $A_{2 h}$ is symmetric.

\section{NUMERICAL EXPERIMENTS}

The multigrid schedule used is the $W$-cycle with one post-smoothing iteration. The smoothing method is the $I L U$-method described in Wesseling $(1982,1987)$.

The test problems are the interface problems sketched in Fig. 4.1. In the first problem we have two concentric squares, in the second problem the inner square is rotated over $45^{\circ}$. The sides of the outer square have length 1 , of the inner cell $n h$ in problem 1 , and $n h / \sqrt{2}$ in problem 2. In the inner square we have $a=a_{1}=$ $0.333 * 10^{5}$, in the outer square $a=a_{2}=2$. Problem 3 was suggested by Achi Brandt. The cells with centers at $x=(n-1 / 2) h$ constitute a vertical isolating strip of width $h$, where the value of the diffusion coefficients is $a=a_{1}=10^{-10}$; outside the strip, $a=a_{2}=2$. 

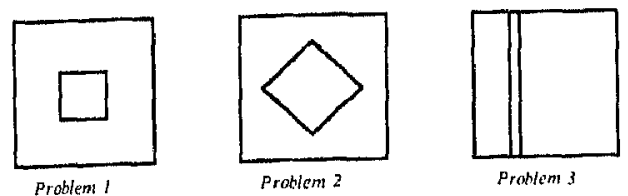

FIG. 4.1. Geometry of test problems.

We solve (1.1) with $f=x y, g=x^{2}+y^{2}$, starting iterand zero. Twelve iterations were carried out. The average reduction factor $\rho$ is defined as

$$
\rho=\left\{\left\|r^{m}\right\| /\left\|r^{o}\right\|\right\}^{1 / m}
$$

with $\|\cdot\|$ the $l_{2}$-norm, $r$ the residue $r=b^{h}-A_{h} \phi^{h}$ on the finest grid (with $A_{h} \phi^{h}=b^{h}$ the system top be solved), $r^{\circ}$ the initial residue, $r^{m}$ the final residue, and $m$ the number of multigrid iterations carried out. Table I gives $\rho$ for a number of cases. Where $n \neq 0$ we have taken the worst case for all $0 \leqslant n \leqslant h^{-1}$. The last column is for Neumann boundary conditions along $x=0$ and $y=0$.

It is clear that multigrid works efficiently. For problems 1 and $2, \rho$ does not increase with $h$. With $n \neq 0, \rho$ is larger than with $n=0$ (Poisson equation). We think this is due to the fact that the equations in the inner square are almost uncoupled from those outside for $a_{1} \gg a_{2}$, so that we almost have a discretized pure Neumann problem for the interior square, which is singular. This hypothesis is confirmed by the fact that with $a_{1}$ and $a_{2}$ interchanged $\left(a_{1} \ll a_{2}\right), \rho$ is found to be about the same size for all $n$, including 0 . For problem $3, \rho$ increases with $h$ for certain locations of the isolating strip. This is thought to be due to the fact that, as suggested by Achi Brandt, according to Eq. (3.13) the isolation (small value of $w$ ) between the regions separated by the vertical strip may disappear after two coarsenings: Nevertheless, convergence is still rapid. Inspection of the last column of Table I shows that the introduction of Neumann boundary conditions has little influence. Therefore it does not seem worthwhile to abandon $(3.8)$ along nonDirichlet boundaries.

With another smoothing method, namely point Gauss Seidel, similar results were obtained.

TABLE I

$n, \rho$ for Problems 1,2 , and 3

\begin{tabular}{|c|c|c|c|c|c|c|c|c|c|c|}
\hline Problem $\backslash h^{-1}$ & & 8 & & 6 & & 2 & & 14 & & 4 \\
\hline 1 & 0 & 0.059 & 0 & 0.077 & 0 & 0.085 & () & $(0)(k) 1$ & 11 & 0090 \\
\hline 1 & 6 & 0.312 & 10 & 0.362 & 26 & 0.304 & $5 x$ & 0.2911 & $5 x$ & 1).29x \\
\hline 2 & 6 & 0.245 & 10 & 0.300 & 26 & 0.273 & 58 & 0.237 & $\zeta k$ & 11.281 \\
\hline 3 & 1 & 0.061 & 10 & 0.074 & 18 & 0.147 & 34 & 11244 & 34 & 0.372 \\
\hline
\end{tabular}




\section{DISCUSSION}

Multigrid methods that work for elliptic equations with discontinuous coefficients (interface problems) have been described by Alcouffe et al. [1], Dendy [3], Kettler and Meijerink [6], Kettler [7], and in the present work. The present method differs from the earlier ones in that grid coarsening is done cell-wise rather than point-wise, and prolongation and restriction are not dependent on the equations. As a result, the present method is simpler and requires less storage.

Comparing the rates of convergence that are reported one gets the impression that the present method is at least as efficient as the earlier ones.

Thanks to the simplicity of the present method, it can be jusified theoretically. The theory will be given elsewhere. Why does the present method work? An important factor probably is that (3.12) is quite similar to (2.13). This suggests that the present prolongation and restriction result in accurate coarse grid approximation. Also, the similarity between (3.12) and (2.13) simplifies the theory.

Extension to 3D seems easier than for the older methods. The same considerations as for the older methods are expected to apply to the extension to systems of differential equations.

\section{ACKNOWLEDGMENTS}

The author is indebted to C. Cuvelier, J. van Kan, and P. Sonneveld for useful discussions.

\section{REFERENCES}

1. R. E. Alcouffe, A. Branit, J. E. Dendy, Jr., and J. W. Painter, SiAM J. Sci. Stat. Comp. 2, 430 (1981).

2. A. Brandt, Math. Compul. 31, 333 (1977).

3. J. E. Dendy Jr., J. Comput. Phys. 48, 366 (1982).

4. W. Hack busch and U. Trottenberg (Eds.), Proceedings, Köln-Porz, Lecture Notes in Mahematics Vol. 960 (Springer-Verlag, Berlin, 1982).

5. W. HaCKBusCH, Multi-Grid Methods and Applications (Springer-Verlag, Berlin, 1985).

6. R. Kettler and J. A. Mejuerink, A Multigrid Method and a Combined Multigrid-Conjugate Gradient Method for Elliptic Problems with Strongly Discontinuous Coefficients in General Domains, SHELL Publ. 604 (KSEPL, Rijswijk, The Netherlands, 1981).

7. R. Kettler, Proceedings, Köln-Porz, edited by Hackbusch and Trottenberg, Lecture Notes in Math. Vol. 960 (Springer-Verlag, Berlin, 1982), pp. 502-534.

8. S. MCCormick (Ed.), Multigrid Methods, Frontiers in Applied Mathematics Vol. 5 (SIAM, Philadelphia, 1987).

9. P. Wesseling, Proceedings, Köln-Porz, edited by Hackbusch and Trottenberg, Lecture Notes in Math. Vol 960 (Springer-Verlag, Berlin, 1982), pp. 164-184.

10. P. Wesseling, Frontiers in Appl. Math. Vol. 5, edited by McCormick (SIAM, Philadelphia, 1987). 